\title{
MAJOR SIMPLIFICATIONS IN A CURRENT LINEAR MODEL FOR THE MOTION OF A THERMOELASTIC PLATE
}

\author{
BY \\ J. G. SIMMONDS \\ University of Virginia, Charlottesville, VA
}

Dedicated to the memory of Warner T. Koiter (1914-1997), friend and mighty mechanician

\begin{abstract}
A dynamic model for a thin thermoelastic plate proposed by Lagnese and Lions in 1988 [1] has been used recently by several authors (e.g., [2]-[5]) to study existence and stability of solutions to initial/boundary-value problems. Simple, systematic orderof-magnitude arguments show that it is consistent to neglect several terms appearing in the governing differential equations that couple a temperature moment to the average vertical displacement. Further, because the time scale on which the temperature adjusts itself to the strain rate contribution to the energy equation is quite small compared with the longest (isothermal) period of free vibration of the plate, the energy equation can be solved for the temperature in terms of derivatives of the vertical displacement and hence the system reduced to a single equation, only slightly more complicated than the classical (Kirchhoff) equation of motion. Among other things, it is shown that the temperature has a cubic rather than a linear variation through the thickness. Finally, another order-of-magnitude estimate for a clamped aluminum plate of one meter radius and $1 \mathrm{~mm}$ thickness shows that thermal damping acting alone takes on the order of 200 cycles of vibration to halve the initial amplitude.
\end{abstract}

1. The equations of linear thermoelasticity. Let $x_{\alpha}, \alpha=1,2$, and $z$ denote Cartesian coordinates in an inertial reference frame and let a homogeneous, isotropic, thermoelastic plate-like body occupy the closure of the open set $\Omega \times(-H, H)$, where $\Omega$, the midplane of the plate, is a connected region of the $x_{\alpha}$-plane with piecewise smooth boundary $\partial \Omega$ and $2 H$ is the plate's thickness. Then, from the standard references by Boley and Weiner [6] or Carlson [7], the linearized governing three-dimensional equations in Cartesian tensor notation comprise the equations of motion,

$$
\begin{gathered}
\sigma_{\alpha \beta, \alpha}+\tau_{\beta, z}+f_{\beta}=\rho \ddot{u}_{\beta}, \\
\tau_{\alpha, \alpha}+\sigma_{, z}+f=\rho \ddot{u}
\end{gathered}
$$

Received November 10, 1997.

1991 Mathematics Subject Classification. Primary 35B20, 73B30.

(C)1999 Brown University 
the energy equation,

$$
k\left(\vartheta_{, \alpha \alpha}+\vartheta_{, z z}\right)+m \theta_{0}\left(u_{\alpha, \alpha}+u_{, z}\right) \cdot r=c \dot{\vartheta},
$$

and the stress-displacement-temperature relations

$$
\begin{gathered}
\sigma_{\alpha \beta}=\mu\left(u_{\alpha, \beta}+u_{\beta, \alpha}\right)+\lambda\left(u_{\gamma, \gamma}+u_{, z}\right) \delta_{\alpha \beta}+m \vartheta \delta_{\alpha \beta}, \\
\tau_{\alpha}=\mu\left(u_{\alpha, z}+u_{, \alpha}\right), \quad \sigma=2 \mu u_{, z}+\lambda\left(u_{\alpha, \alpha}+u_{, z}\right)+m \vartheta .
\end{gathered}
$$

Here $\left(\sigma_{\alpha \beta}, \tau_{\alpha}, \sigma\right)$ are the physical (Cartesian) components of the stress tensor, $\left(f_{\alpha}, f\right)$ are the components of the body force vector, $\rho$ is the (constant) mass per unit volume, $\left(u_{\alpha}, u\right)$ are the components of the displacement vector, $k$ is the thermal conductivity, $\vartheta$ is the increment in absolute temperature above the constant reference value $\theta_{0}, m$ is the stresstemperature modulus, $r$ is the heat supply, $c$ is the specific heat, and $\lambda$ and $\mu$ are the Lamé elastic constants. Further, a subscript preceded by a comma denotes differentiation with respect to that subscript and a dot denotes differentiation with respect to time. For simplicity, we shall henceforth take $f_{\alpha}=f=r=0$.

To make our expansion procedure easier, we rewrite (1.5) in the inverted form

$$
u_{\alpha, z}=-u_{, \alpha}+(1-\nu)\left(\tau_{\alpha} / E\right)
$$

and

$$
(1-\nu) u_{, z}=-\nu u_{\alpha, \alpha}+(1+\nu)(1-2 \nu)(\sigma / E)+(1+\nu) \alpha \vartheta
$$

where $E$ is Young's modulus, $\nu$ is Poisson's ratio, and

$$
\alpha=-\frac{m}{3 \lambda+2 \mu}=-\frac{1-2 \nu}{E} m
$$

is the coefficient of thermal expansion. We then substitute (1.7) into (1.3) and (1.4), so obtaining

$$
k\left(\vartheta_{, \alpha \alpha}+\vartheta_{, z z}\right)=\frac{E \theta_{0} \alpha}{1-\nu}\left(u_{\alpha, \alpha}+\frac{1+\nu}{E} \sigma\right)+c^{*} \dot{\vartheta}
$$

where

$$
c^{*}=c+\frac{(1+\nu) E \theta_{0} \alpha^{2}}{(1-\nu)(1-2 \nu)}
$$

is a modified specific heat, and

$$
\sigma_{\alpha \beta}=\frac{E}{1-\nu^{2}}\left[\nu \delta_{\alpha \beta} u_{\gamma, \gamma}+\frac{1}{2}(1-\nu)\left(u_{\alpha, \beta}+u_{\beta, \alpha}\right)-(1+\nu) \alpha \vartheta \delta_{\alpha \beta}\right]+\frac{\nu}{1-\nu} \sigma \delta_{\alpha \beta} .
$$

In view of (1.11), our basic unknowns are now the displacement components, $\left(u_{\alpha}, u\right)$, the transverse and normal stresses, $\left(\tau_{\alpha}, \sigma\right)$, and the incremental temperature, $\vartheta$.

At the faces of the plate, we shall, for simplicity, assume that there are no surface tractions, i.e.,

$$
\tau_{\alpha}\left(x_{\alpha}, \pm H, t\right)=\sigma\left(x_{\alpha}, \pm H, t\right)=0,
$$

and that Newton's Law of cooling holds, i.e.,

$$
\vartheta_{, z}\left(x_{\alpha}, \pm H, t\right) \pm b \vartheta\left(x_{\alpha}, \pm H, t\right)=0
$$


where $b$ is a radiation coefficient. (This is the same thermal boundary condition assumed in [1].)

2. Scaling of variables. Let $L$ be the radius of the largest disk contained in the closure of the midplane of the plate and introduce dimensionless spatial variables by setting

$$
x_{\alpha}=L \xi_{\alpha}, \quad z=H \zeta .
$$

Classical plate theory is based on the premise that differentiation of any field variable with respect to $\xi_{\alpha}$ or $\zeta$ does not change its order of magnitude. As is well known, this assumption breaks down in the vicinity of edges or concentrated loads where there exist three-dimensional boundary layers of width $O(H)$.

To derive classical thermoelastic plate theory in a systematic (if formal) way, we must introduce an appropriate scaling of the displacement, stress, time, and temperature variables. To this end let $w_{0}$ denote a reference vertical displacement of the midplane of the plate. Then to guarantee that our formal expansion procedure will yield the classical Kirchhoff equation for static isothermal deformation, we introduce dimensionless displacements by setting

$$
u_{\alpha}=(H / L) w_{0} U_{\alpha}, \quad u=w_{0} U
$$

The stresses are scaled in the standard way:

$$
\tau_{\alpha}=\left(E H^{2} w_{0} / L^{3}\right) T_{\alpha}, \quad \sigma=\left(E H^{3} w_{0} / L^{4}\right) S .
$$

In scaling the time variable, we note that there exist three disparate time scales: (1) the time it takes any transient initial temperature distribution to decay to, say, half its initial value; (2) the period of the lowest natural isothermal mode of vibration; and (3) the time it takes for any initial motin of the plate to decay to, say, half its initial value. Since (2) is much greater than (1) and much less than (3), we choose the former to nondimensionalize the time. To determine this time scale, we turn to the classical isothermal equation of motion of a plate,

$$
D w_{, \alpha \alpha \beta \beta}+2 H \rho \ddot{w}=0,
$$

where

$$
D=\frac{2 E H^{3}}{3\left(1-\nu^{2}\right)}
$$

is the plate's bending stiffness and $w$ is some measure of the vertical deflection. In accordance with the scaling (2.1), the lowest natural frequency of vibration of an isothermal plate is $O\left[\left(H / L^{2}\right) \sqrt{E / \rho}\right]$. Thus, we introduce a dimensionless time by setting

$$
t=\left(L^{2} / H\right) \sqrt{\rho / E} \tau .
$$

To choose an appropriate scale for the temperature increment $\vartheta$, we simply require that the temperature effect in the stress-displacement-temperature relation (1.11) be significant. This leads to the nondimensionalization

$$
\vartheta=\left(H w_{0} / \alpha L^{2}\right) \Theta
$$


3. The scaled governing equations. We shall rewrite the governing equations in the order we shall integrate them. Thus, with the scaled variables defined in $(2.1)-(2.3)$, (2.6), and (2.7), with $f_{\text {. }}$ now denoting $\partial f / \partial \xi_{\alpha}$, and with

$$
\varepsilon=H / L
$$

denoting the fundamental small parameter of plate theory, we find that $(1.7),(1.6),(1.9)$, (1.1), and (1.2) can be written

$$
\begin{gathered}
U_{, \zeta}=O\left(\varepsilon^{2}\right), \\
U_{\alpha, \zeta}=-U_{. \alpha}+O\left(\varepsilon^{2}\right), \\
\Theta_{, \zeta \zeta}+O\left(\varepsilon^{2}\right)=\left\{\beta\left[U_{\alpha . \alpha}+O\left(\varepsilon^{2}\right)\right]+\gamma \Theta\right\}_{, \hat{\tau}}, \\
\left(1-\nu^{2}\right) T_{\beta, \zeta}=-\left[\frac{1}{2}(1+\nu) U_{\alpha . \alpha \beta}+\frac{1}{2}(1-\nu) U_{\beta, \alpha \alpha}-(1+\nu) \Theta_{. \beta}\right]\left[1+O\left(\varepsilon^{2}\right)\right], \\
S_{. \zeta}=U_{, \tau \tau}-T_{\alpha, \alpha} .
\end{gathered}
$$

In $(3.4)$,

$$
\beta=\frac{E^{3 / 2} \theta_{0} \alpha^{2} L}{(1-\nu) \rho^{1 / 2} k} \quad \text { and } \quad \gamma=\frac{E^{1 / 2} c^{*} L}{\rho^{1 / 2} k}
$$

are dimensionless thermal constants that are independent of the plate's thickness and

$$
\hat{\tau}=\tau / \varepsilon^{3}
$$

is a "fast" time variable.

Finally, we note by $(2.1),(2.3)$, and (2.7) that the homogeneous face boundary conditions (1.12) and (1.13) take the form

$$
T_{\alpha}\left(\xi_{\alpha}, \pm 1, \tau\right)=S\left(\xi_{\alpha}, \pm 1, \tau\right)=0
$$

and

$$
\Theta_{. \zeta}\left(\xi_{\alpha}, \pm 1, \tau\right) \pm \varepsilon b L \Theta\left(\xi_{\alpha}, \pm 1, \tau\right)=0 .
$$

4. The equations for the flexure of a classical thermoelastic plate. By the flexural motion of a plate, we mean solutions of (3.2)-(3.6) such that

$$
\left\{\begin{array}{c}
U, T_{\alpha} \\
U_{\alpha}, \sigma, \Theta
\end{array}\right\} \text { are }\left\{\begin{array}{c}
\text { even } \\
\text { odd }
\end{array}\right\} \text { in } \zeta .
$$

By the classical theory of flexure of a thermoelastic plate we mean Eqs. (3.2)-(3.6) and the associated face conditions in the limit as $\varepsilon \rightarrow 0$. Thus, (3.2), (3.3), and (4.1) yield

$$
U=w\left(\xi_{\alpha}, \tau\right), \quad U_{\alpha}=-\xi w_{. \alpha}\left(\xi_{\alpha}, \tau\right),
$$

the fields one often assumes a priori under the Kirchhoff Hypothesis. Here they fall out as a consequence of our scaling and the assumption that differentiation with respect to $\xi_{\alpha}$ or $\zeta$ does not change orders of magnitude. Substituting $(4.2)_{2}$ into (3.4), we have, in the limit as $\varepsilon \rightarrow 0$,

$$
\Theta_{. \zeta \zeta}=\left(\gamma \Theta-\beta \zeta w_{, \alpha \alpha}\right)_{, \hat{\tau}} .
$$


The solution of this equation, odd in $\zeta$, is subject to the limiting form of the boundary condition (3.10), namely

$$
\Theta_{, \zeta}\left(\xi_{\alpha}, 1, \tau\right)=0
$$

together with an initial condition of the form

$$
\Theta\left(\xi_{\alpha}, \zeta, 0\right)=\widetilde{\Theta}\left(\xi_{\alpha}, \zeta\right)
$$

where $\widetilde{\Theta}$ is prescribed.

Using the odd eigenfunctions $\sin \lambda_{n} \zeta, \lambda_{n}=\frac{1}{2} \pi+n \pi, n=0,1, \ldots$, associated with the operator $-d^{2} / d \zeta^{2}$ and the boundary condition (4.4), we find that the solution of (4.3) satisfying (4.4) and (4.5) can be expressed in the form

$$
\Theta=\sum_{n=0}^{\infty}\left[e^{-\left(\lambda_{n}^{2} / \gamma\right) \hat{\tau}} \widetilde{\Theta}_{n}\left(\xi_{\alpha}\right)+(2 \beta / \gamma)(-1)^{n} \lambda_{n}^{-2} \int_{0}^{\hat{\tau}} e^{-\left(\lambda_{n}^{2} / \gamma\right)(\hat{\tau}-\bar{\tau})} w_{. \alpha \alpha \hat{\tau}} d \bar{\tau}\right] \sin \lambda_{n} \zeta
$$

where

$$
\widetilde{\Theta}_{n}=2 \int_{0}^{1} \widetilde{\Theta}\left(\xi_{\alpha}, \zeta\right) \sin \lambda_{n} \zeta d \zeta
$$

If we formally introduce the fast time (3.8) as a second time variable, then (3.6) and (4.2) imply that

$$
w_{. \hat{\tau} \hat{\tau}}=O\left(\varepsilon^{3}\right) \quad \text { or } \quad w_{, \hat{\tau}}=w_{, \hat{\tau}}\left(\xi_{\alpha}, \tau\right)\left[1+O\left(\varepsilon^{3}\right)\right]
$$

which means that, within the framework of classical plate theory, (4.6) may be given the explicit form

$$
\begin{aligned}
& \Theta=\frac{1}{2} \zeta\left(1-\zeta^{2} / 3\right) \beta w_{, \alpha \alpha \hat{\tau}}\left(\xi_{\alpha}, \tau\right) \\
& \quad+\sum_{n=0}^{\infty} e^{-\left(\lambda_{n}^{2} / \gamma\right) \hat{\tau}}\left[\widetilde{\Theta}_{n}\left(\xi_{\alpha}\right)-2 \beta(-1)^{n} \lambda_{n}^{-4} w_{, \alpha \alpha \hat{\tau}}\left(\xi_{\alpha}, \tau\right)\right] \sin \lambda_{n} \zeta .
\end{aligned}
$$

As $\hat{\tau} \rightarrow \infty$ or, from the viewpoint we prefer when deriving classical plate theory, as $\varepsilon \rightarrow 0$ for $\tau$ fixed, $\Theta$ approaches the first line on the right of (4.9). This limit also follows if we ignore the first term on the right of (4.3) and means physically that any initial temperature distribution is equilibrated virtually instantaneously on a time scale of the order of the longest period of oscillation of a classical isothermal plate. Henceforth, we replace $\Theta$ by this limit which we note is cubic in $\zeta$ rather than linear, as is assumed in [1].

Substituting $\Theta=\frac{1}{2} \zeta\left(1-\zeta^{2} / 3\right) \beta w_{, \alpha \alpha \hat{\tau}}$ along with $(4.2)_{2}$ into the limiting form of (3.5), we have

$$
\left(1-\nu^{2}\right) T_{\beta, \zeta}=\zeta\left[w+\frac{1}{2}(1+\nu) \beta\left(1-\zeta^{2} / 3\right) w_{, \hat{\tau}}\right]_{, \alpha \alpha \beta} .
$$

Integrating (4.10) with respect to $\zeta$ and applying the boundary condition $(3.9)_{1}$, we obtain

$$
\left(1-\nu^{2}\right) T_{\beta}=\frac{1}{2}\left(\zeta^{2}-1\right)\left[w+\frac{1}{12}(1+\nu)\left(5-\zeta^{2}\right) w_{, \hat{\tau}}\right]_{, \alpha \alpha \beta}
$$

Our final equation to integrate is (3.6) which, by $(4.2)_{1}$ and (4.11), now reads

$$
\left(1-\nu^{2}\right) S_{, \zeta}=\left(1-\nu^{2}\right) w_{, \tau \tau}+\frac{1}{2}\left(1-\zeta^{2}\right)\left[w+\frac{1}{12}(1+\nu) \beta\left(5-\zeta^{2}\right)\right]_{, \alpha \alpha \beta \beta} .
$$


On integrating both sides with respect to $\zeta$ from 0 to 1 and imposing the boundary condition $(3.9)_{2}$, we obtain a single dimensionless partial differential equation for the motion of a thermoelastic plate:

$$
\left[w+\frac{2}{5}(1+\nu) \beta w_{, \hat{\tau}}\right]_{, \alpha \alpha \beta \beta}+3\left(1-\nu^{2}\right) w_{, \tau \tau}=0 .
$$

The stress couples and transverse shear stress resultants (needed to satisfy any prescribed edge loading) follow from (1.11), (2.2), (2.3), (2.6), (2.7), (3.1), (4.1), (4.2), (4.11), and the first line of (4.9) as

$$
M_{\alpha \beta}=-\int_{-H}^{H} z \sigma_{\alpha \beta} d z=\left(D w_{0} / L^{2}\right)\left[\nu \delta_{\alpha \beta} w_{, \gamma \gamma}+(1-\nu) w_{, \alpha \beta}\right]
$$

and

$$
Q_{\beta}=\int_{-H}^{H} \tau_{\beta} d z=-\left(D w_{0} / L^{3}\right) w_{, \alpha \alpha \beta},
$$

where $O\left(\varepsilon^{2}\right)$ terms have been dropped.

5. Effect of thermal damping on natural modes of vibration. To illustrate the effect of thermal damping on the modes of vibration of a thin plate, we consider a plate clamped along its boundary, i.e.,

$$
w=w_{, \alpha} \nu_{\alpha}=0 \quad \text { on } \partial \Omega,
$$

where $\nu_{\alpha}$ are the Cartesian components of the outward unit normal to $\partial \Omega$. Let $\phi_{n}\left(\xi_{\alpha}\right)$ be the corresponding $n$th dimensionless natural mode of vibration according to isothermal Kirchhoff plate theory, i.e.,

$$
\phi_{n, \alpha \alpha \beta \beta}=3\left(1-\nu^{2}\right) \omega_{n}^{2} \phi_{n},
$$

where $\left(H / L^{2}\right) \sqrt{E / \rho} \omega_{n}$ is the corresponding natural frequency of vibration. Then it is clear that (4.13), subject to the homogeneous boundary conditions (5.1), admits solutions of the form

$$
w=e^{p \tau} \phi_{n}\left(\xi_{\alpha}\right), \quad p=\text { constant } .
$$

Substituting (5.3) into (4.13), using (3.8) to replace the fast time variable by the dimensionless time $\tau$, and noting (5.2), we obtain the characteristic equation

$$
p^{2}+\frac{2}{5}(1+\nu) \beta \varepsilon^{3} \omega_{n}^{2} p+\omega_{n}^{2}=0
$$

whose solution has the form

$$
p=-\left(\varepsilon^{3} / 5\right)(1+\nu) \beta \omega_{n}^{2} \pm i\left[1+O\left(\varepsilon^{6}\right)\right] \omega_{n} .
$$

[We also could have obtained this result-but with more labor-by introducing a "slow" time variable $\tilde{\tau}=\varepsilon^{3} \tau$ and using the two-scale method to find an asymptotic solution of (4.13) of the form $w(\tau, \tilde{\tau} ; \varepsilon)=\stackrel{\circ}{w}(\tau, \bar{\tau})+O\left(\varepsilon^{3}\right)$.] Thus, with those real parts of $p$ neglected that are of relative order $\varepsilon^{6},(5.3)$ reads

$$
w=e^{-\left(\varepsilon^{3} / 5\right)(1+\nu) \beta \omega_{n}^{2} \tau} e^{ \pm i \omega_{n} \tau} \phi_{n}\left(\xi_{\alpha}\right)
$$


which shows that the ratio $R$ of the natural period of vibration to the time it takes $w$ to decay to half its initial amplitude is

$$
R=\left(2 \pi \varepsilon^{3} /(5 \ln 2)\right)(1+\nu) \beta \omega_{n} .
$$

For a clamped circular aluminum plate of one meter radius and one millimeter thickness vibrating in a vacuum at room temperature, we find, using the elastic and thermal constants given on pp. 87 and 157 of [8] and the natural frequencies of vibration given in Table 2.1 of [9], that $R$ for the lowest natural frequency of vibration is about $4.84 \times 10^{-3}$. Since the lowest natural period of vibration works out to about 0.075 seconds, this means that it takes about 15 seconds for the amplitude of vibration of the plate to damp to half its initial value.

Acknowledgment. This research was supported by the U. S. Army Research Office under Grant DAAH04-94-G0189 and by the National Aeronautics and Space Administration under Grant NAG-1-1854.

\section{REFERENCES}

[1] J. Lagnese and J.-L. Lions, Modelling Analysis and Control of Thin Plates, Recherches en Mathématiques Appliquées (P. G. Ciarlet and J.-L. Lions, Eds.), Masson, Paris, 1988

[2] J. Lagnese, Boundary Stabilization of Thin Plates, SIAM, Philadelphia, 1989

[3] J. Lagnese, The reachability problem for thermoelastic plates, Arch. Rat. Mech. Anal. 112, 223-267 (1990)

[4] J. U. Kim, On the energy decay of a linear thermoelastic bar and plate, SIAM J. Math. Anal. 23, 889-899 (1992)

[5] Z. Liu and S. Zheng, Exponential stability of the Kirchhoff plate with thermal or viscoelastic damping, Quart. Appl. Math. LV, 551-564 (1997)

[6] B. A. Boley and J. H. Weiner, Theory of Thermal Stresses, Krieger, Malabar, FL, 1985

[7] D. E. Carlson, Linear thermoelasticity, Mechanics of Solids, Vol. II (C. Truesdell, Ed.), SpringerVerlag, Berlin, 1984, pp. 297-345

[8] T. H. Dawson, Theory and Practice of Solid Mechanics, Plenum Press, New York, 1976

[9] A. W. Leissa, Vibration of Plates, NASA SP-160, 1969 